To the Editors:

\title{
Takotsubo cardiomyopathy after an emotional outburst
}

\author{
N L A Shyamali ${ }^{1}$, H Ranawaka ${ }^{2}$ \\ Ceylon Medical Journal 2015; 60: 113-114
}

Takotsubo cardiomyopathy (TTC) is characterised by transient systolic dysfunction of the apical and midsegment of the left ventricle with apical ballooning in the absence of obstructive coronary artery lesions [1]. It is usually preceded by severe stress and the clinical presentation is similar to acute coronary syndrome.

A 63-year old female was admitted with sudden unbearable retrosternal chest pain which started immediately after a heated argument with her daughterin-law. There were no cardiovascular risk factors. Examination was normal and electrocardiogram (ECG) showed ST elevation in leads I, aVL, and V6 and ST depression with $\mathrm{T}$ inversion in lead III, aVF (Figure). Troponin I was $4.43 \mathrm{ng} / \mathrm{ml}$ (normal $<0.04 \mathrm{ng} / \mathrm{ml}$ ) and other investigations were normal.

Echocardiography showed severely hypokinetic mid and apical segments of left ventricle (LV) with apical ballooning and poor LV function of $30 \%$. Cardiac catheterisation revealed normal coronary vasculature. She was started on oral carvedilol $3.125 \mathrm{mg}$ twice a day, ramipril $2.5 \mathrm{mg}$ once a day, frusemide $20 \mathrm{mg}$ twice a day and aspirin $75 \mathrm{mg}$ once a day. Her exercise tolerance improved gradually and she was completely asymptomatic after four weeks. Repeat echocardiography showed remarkable improvement in the LV systolic function with a normal ejection fraction and normal wall motion.

Although this case illustrates typical features of TTC after severe emotional distress, it can also be triggered by physically stressful events [1]. Emotional stressors range from a discrete event such as receipt of bad news to an ongoing relationship problem. Physical stressors typically involve trauma, major surgery or any severe illness including sepsis [1]. The pathophysiology remains unclear, but catecholamine-induced microvascular spasm or dysfunction, leading to myocardial stunning is the most favored explanation [2,3].

TTC was first described in 1991 in Japan and named takotsubo-like LV dysfunction in reference to the associated LV morphological features on left ventriculography. "Tako tsubo" is a pot with a round bottom and narrow neck used for trapping octopuses in Japan [4]. The incidence is $1-2 \%$ of all cases of suspected acute

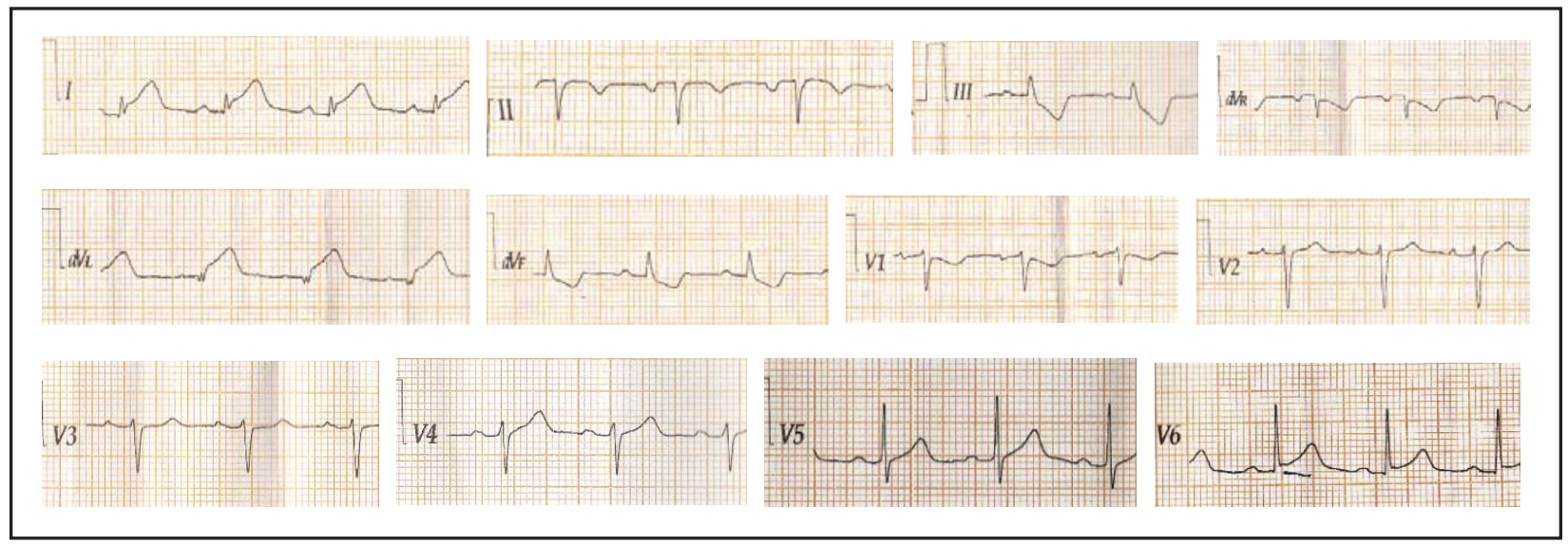

Figure. Electrocardiogram showing ST elevation and ST depression with T inversion.

${ }^{1}$ Department of Medicine, Faculty of Medical Sciences, University of Sri Jayewardenepura and ${ }^{2}$ Department of Cardiology, Dr. Neville Fernando Teaching Hospital, Sri Lanka.

Correspondence: NLAS, e-mail: <ajaliyanage@yahoo.com>. Received 11 February and revised version accepted 18 April 2015. 
myocardial infaraction (MI). TTC has a striking female predominance, reportedly almost $90 \%$ of cases. Affected women are typically postmenopausal, around the age of 60-76 years $[1,2,5]$.

Onset of this condition is abrupt and unpredictable. The frequent presenting symptoms are chest pain and dyspnea, resembling acute MI [3,5]. Other clinical presentations include syncope, shock and arrhythmia. The most common ECG changes are ST elevation and T inversion. Prolongation of QT interval and abnormal Q waves have been reported. In some cases ECG can be normal [2]. Echocardiography or ventriculography reveal left mid-ventricular dysfunction and apical dyskinesis or akinesis with apical ballooning. The mean LV Function ranges from $20-49 \%$. A normal coronary angiography is required in the acute setting to confirm the diagnosis. However mild, non-obstructive coronary lesions ( $<50 \%$ luminal diameter stenosis) might be present. Serum catecholamines and brain natriuretic peptide (BNP) levels are usually high. Cardiac enzyme levels are increased $[2,3,5]$. Peak troponin levels are modest, mean $\sim 60$-fold as opposed to $>400$-fold rise for acute MI [1]. Modified Mayo Clinic diagnostic criteria are widely used in clinical practice and research for diagnosis of TTC [1].

Supportive therapy with standard drugs for LV failure is recommended as wall-motion abnormalities are completely resolved and LV function is normalised within a few weeks $[2,5]$. The prognosis is excellent, and recurrences occur in $<10 \%$ of patients [3]. Repeat echocardiography demonstrates recovery of LV function. Clinicians need to be aware of the existence and manifestations of TTC which should be suspected in patients with features suggestive of acute coronary syndrome after an stressful event.

\section{References}

1. Scantlebury DC, Prasad A. Diagnosis of Takotsubo Cardiomyopathy - Mayo Clinic Criteria. Circulation $J$ 2014; 78: 2129-39.

2. Gianni M, Dentali F, Grandi A, Sumner G, Hiralal R, Lonn E. Apical ballooning syndrome or tako-tsubo cardiomyopathy: a systematic review. Eur Heart J 2006; 27: 1532-43.

3. Prasad A, Lerman A, Rihal C. Apical ballooning syndrome (Takotsubo or stress cardiomyopathy): a mimic of acute myocardial infarction. Am Heart J 2008; 155: 408-13.

4. Dote K, Sato H, Tateishi H, Uchida T, Ishihara M. Myocardial stunning due to simultaneous multivessel coronary spasms: a review of 5 cases. J Cardiol 1991; 21: 203-14.

5. Akashi Y, Goldstein D, Barbaro G, Ueyama T. Takotsubo cardiomyopathy: a new form of acute, reversible heart failure. Circulation 2008; 118: 2754-9. 